\title{
Gender Classification using Face Images: A Review
}

\author{
Dhanashri P. Lale \\ Department of Electronics and Telecommunication Engineering, \\ SKN Sinhgad college of Engineering, Pandharpur \\ Tal- Pandharpur. Dist- Solapur. State- Maharashtra. India. Pin code- 413304 \\ Kailash J. Karande \\ Department of Electronics and Telecommunication Engineering, \\ SKN Sinhgad college of Engineering, Pandharpur \\ Tal- Pandharpur. Dist- Solapur. State- Maharashtra. India. Pin code- 413304
}

\begin{abstract}
In field of Image processing face is one of the most important biometric traits and is becoming more popular for the security purpose in now a days. During past several years classification of gender from facial images has gained enormous significance and has become a popular area of research. Many researches have done on the gender classification from several years. Still, this is a very important field of image processing because of its applications in many areas like monitoring, surveillance, commercial profiling and human-computer interaction. Security applications have high importance in this area. Gender classification using facial and racial features can be used as part of a face recognition process. This paper comparison of different gender classification techniques and use of different racial features such as eyes, nose, mouth etc. for gender classification.
\end{abstract}

Keywords - Gender Classification; facial and racial features extraction.

\section{INTRODUCTION}

Identifying gender, age and ethnicity of human faces [7] using computer has been popular and gaining immense attention in past several years. These attributes such as gender, age, and ethnicity can play an important role in many applications such as human-computer interaction, surveillance, content-based indexing and searching, biometrics, demographic studies and targeted advertising. Studies have shown that a human can easily differentiate between a male and female but, it is a difficult for machine. There exist some distinguishable features between male and female which are used by machine to classify gender. Gender recognition is a pattern recognition problem [14]. Pattern recognition can be divided into two parts, one and two stage pattern recognition systems. One stage pattern recognition system classifies input data directly. Two stage pattern recognition systems consist of feature extractor, with classifier. In addition to facial features, racial features such as eyes, nose, mouth etc. are also used for gender classification. In artificial intelligence image processing and machine learning plays an very important role and perform difficult jobs such as face recognition, gesture recognition, facial expression recognition and gender classification [9].

Some applications should not require the physical contact or attention of human being. But some applications require human attention or physical contact. Human parts such as iris, hand or fingerprint would require some cooperation from the human and thus limit its applicability. Whereas face is the most acceptable biometric trait than others because it is easily approachable to the machine therefore large no of application areas are identified where gender recognition is very much involved [2]. 


\section{LITERATURE REVIEW}

In 2005, Jain et.al [1] presented an approach using ICA and SVM. The experiment was done with different classifiers namely cosine classifier which find distance between two features lying on a hyper-sphere surface, linear discriminant classifiers that finds the projection of the input image maximizing the ratio between class scatter and within class scatter, and SVM which finds the maximal separating hyper plane between male and female features. The experiment was performed on 500 images from the FERET facial database which included 250 images of female and 250 images of male, and obtained an accuracy of $96 \%$ in ICA space.

Erno Makinen and Roope Raisamo [2] experimented on gender classification with automatically detected and aligned faces. The experimented was performed on IMM database and FERET database with four automatic alignment methods and four different gender classification methods. In Automatic alignment methods, three methods were based on Active Appearance Model and one based on profile alignment. The four Gender classification methods were used. The automatic face alignment methods did not increase the classification rate where as manual alignment increased the classification rate. The classification accuracy was dependent on face image resizing before or after alignment. The best classification rate was obtained with SVM using pixel based input images of size $36 \times$ 36.

Tejas et.al [3] experimented the gender recognition problem with discriminant functions which include PCA, LDA and Subclass Discriminant Analysis on a heterogeneous data base of 8112 images that included variations in illumination, expression, minor pose and ethnicity. The result showed that PCA provides better performance than $\mathrm{PCA}+\mathrm{LDA}, \mathrm{PCA}+\mathrm{SDA}$, and PCA+SVM. The result showed that linear discriminant functions provide good generalization capability with limited of training samples, Principal components.

Method proposed by Samarasena Buchala et.al. [4] PCA encodes face image properties such as gender, ethnicity, age, and identity efficiently - the classification performances on all properties are reasonably high and much above chance levels. Different components of PCA encode different properties of faces. Very few components are required to encode properties such as gender, ethnicity and age and these components are predominantly amongst the first few components which capture large part of the variance of the data. Large number of components is required to encode identity and these components are widely distributed. There may be components which encode multiple properties for example the third component is found to be important for gender as well as age.

F. Saei Manesh, M. Ghahramani, Y. P. Tan [5] experimented "Facial Part Displacement Effect on Template-based Gender and Ethnicity Classification". The gender and ethnicity recognition, by using automatically separated face regions using the modified Golden ratio mask. Facial parts are preprocessed with multiple base point photometric normalization to prevent facial parts displacement in the noted mask, due to different facial parts' distances of people. SVM is employed as the classifier on the extracted Gabor features of each patch to get its confidence level. The final classification results are obtained based on the output of each patch decision using the optimum decision making rule. Finally, using the most accurate normalization approach for each patch, 94\% and 98\% for gender and ethnicity respectively on a dataset composed of FERET and PEAL frontal face images.

Gutta et al.[6] proposed a hybrid classifier based on RBF networks and inductive decision trees for classification of gender and ethnic origin, using a $64 \times 72$ image resolution. The achieved an average accuracy rate of $92 \%$ for the ethnic classification part of the task.

Md. Golam Moazzam et.al. [8] Proposed face recognition using PCA and FLD. The experiment was performed using different levels of additive noise and rotations in handling face recognition problems. The combination of PCA and FLD gives the better results.

Samarasena Buchala et.al. [11] Implemented Global and Feature based Gender Classification of Faces. The experiment was done by using PCA, CCA and SOM. These algorithms were applied to different face parts like eyes, mouth and full face image. The PCA gives $87.5 \%$ result than other methods.

Xiaoguang Lu et.al.[12] proposed "Ethnicity Identification from Face Images" Ethnicity classification was done by using LDA. Different datasets separated into Asian \& Nonasian. Ensemble LDA gives more accuracy than nearest neighbor classifier.

M. Nazir et.al [13] implemented Discrete Cosine Transformation (DCT) technique. Stanford University Medical Student (SUMS) frontal facial images database was used. The KNN classifier using Euclidean distance to find closest neighbors. The ratio of training and testing image is 50 to 50 for KNN classifier. Then the obtainable accuracy is $99.3 \%$. and results are more accurate as compared to support vector machine.

Ziyi Xu et.al.[15] presented a hybrid method of fusing global features and local features. Adaboost algorithm was used to extract global features and AAM was used for local features. The result shows that a hybrid method gives higher accuracy. 
Ravi and Wilson [16] proposed an algorithm converts the RGB image into the $\mathrm{YCbCr}$ color space to detect the skin regions in the facial image. But for getting facial features, they convert RGB images to gray scale image. Authors combined face detection with facial features and gender classification to produces more accurate results; according to them face detection works as pre-processor for classification. The area of skin is detected by converting RGB to gray scale image. Y represents the luminance of skin color while rest represents the color. Features of the face like mouth, lip and eyes are detected after converting the color images into gray scale images. In this experiment Support Vector machine as a gender classifier.

\section{GENERAL SYSTEM}

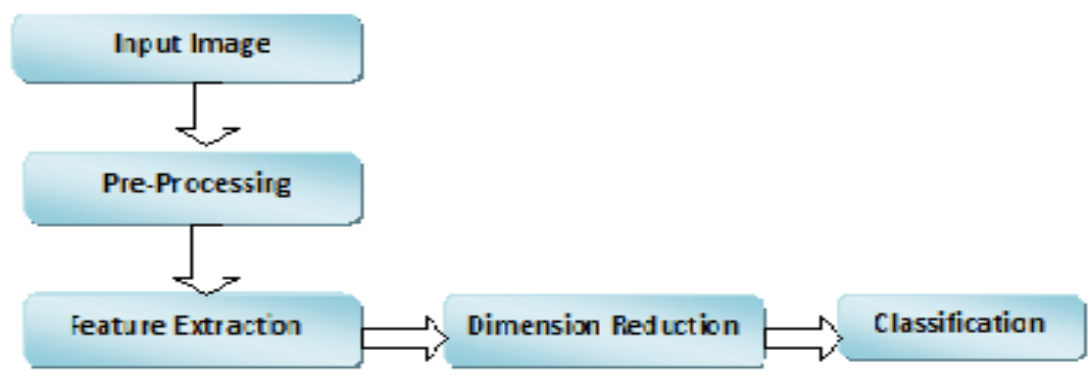

Figure1. General Block Diagram of Gender Classification

Generally gender classification consists of the following steps. Figure 1 depicts these steps.

Pre-Processing: Pre-processing reduces variations like illumination, poses and inaccuracies. Many Classifiers are sensitive to variations. To reduce this sensitivity some pre-processing steps are performed. Some basic Preprocessing steps are involved.

$>$ Facial portion detection and removal of background regions.

$>$ Normalization of brightness using Histogram equalization function.

$>$ Face portion alignments

$>$ Downsizing to reduce the data dimensions

Erno Makinen and Roope Raisamo experiment [2] with four automatic alignment methods, the automatic alignment methods were just not reliable enough. The automatic alignment did not increase the gender classification rates whereas manual alignment actually did increase the classification rates. Ziyi Xu et. al.[15] experimented on gender classification the pre-processing technique was face normalization (some geometric alignment and gray level normalization). Swathi Kalam and Geetha Guttikonda[18] experimented for gender classification and used Preprocessing techniques such as colour conversion, noise reduction, and edge detection.

\section{FEATURE EXTRACTION:}

Feature Extraction is next step of Pre-Processing we need to extract face features. Generally two types of features are extracted namely Geometric based features and appearance based features.

\section{GEOMETRIC-BASED FEATURE EXTRACTION:}

In geometric-based method, features are extracted from some facial points like face, nose and eyes. Loss of useful information takes place using geometric-based techniques Swathi Kalam and Geetha Guttikonda[18] reported 95.6\% accuracy rate after calculating the the ratios of left_eye to right_eye distance, eye to nose, eye to lip and eye to chin distance for feature extraction. Syed Zulqarnain Gilani and Ajmal Mian[20] experimented with 3D Euclidean Distances and for that accuracy rate is $88.36 \%$. Li et al. [19] classified the gender by utilizing not only the five facial features(nose, eyes, mouth, forehead, brows) but also external information like clothes and hair features. Ziyi Xu et. al.[15] used AAM to get 83 landmarks from the face images. 


\section{APPEARANCE-BASED FEATURE EXTRACTION:}

In appearance-based method, features are extracted from the whole face part instead of extracting features from facial points. Colomb et al. [20] reported $91.9 \%$ accuracy after performing experiments on a face database containing 90 images. They used SEXNET network to classify gender. Nazir et al. [13] used discrete cosine transform (DCT) to extract face features. K-nearest neighbor classifier is trained and tested by these features. Experiments are performed on SUMS face database. Problem with their proposed method is that it is not robust to occlusion change. Ravi and Wilson [16] presented a novel gender classification strategy. Face portion is located using skin color and then after extracting geometric-based facial features they applied vector machine to classify gender. Drawback of this method is to choose the correct threshold value for face features extraction. Ziyi Xu et. al.[15] used Adaboost algorithm to extract features.

\section{CLASSIFICATION:}

The last step of gender classification is classification in which the face is successfully classified as that of a male or female. In classification different classifiers are trained and tested by different extracted features. Different classifiers are combined to minimize the classification error rate. These classifiers are called as ensemble classifiers. Combining the outcomes of different classifiers is known as ensemble classifiers. As compare to ensemble classifiers, single classifier accuracy rate is less. E.Makinen et.al [2] used ensemble classifiers such as multilayer Neural Network, Support Vector machine, SVM with LBP features, discrete Adaboost with Haar like Features to increase the classification rate. Nazir et al [13] ensemble different classifiers(i.e. K-nearest neighbour, Mahalanobis distance, Linear Discriminant analysis and kmeans)

\section{IV.CONCLUSION}

Gender classification is done by using gait-based, body-based and face-based. But gender classification using face images is preferred mostly because face is most acceptable biometric trait. In this paper, we have discussed gender classification method uses pre-processing, Feature Extraction and then classification. Working on pixels to classify gender is more expensive so for gender classification, prefers to extract face features rather than direct work on pixels. Feature-based methods are categories into two i.e. global feature and local features.

\section{REFERENCES}

[1] A. Jain, J. Huang, and S. Fang., " Gender identification using frontal facial images”.In IEEE International Conference on Multimedia and Expo, ICME 2005,volume 2005, pages 1082-1085, 2005

[2] E. Makinen and R. Raisamo. "Evaluation of gender classification methods with Automatically detected and aligned faces," IEEE Transactions on Pattern Analysis and Machine Intelligence, 30(3):541-547, 2008.

[3] Tejas, Anush, Richa, Mayank, "Is Gender Classification Across Ethnicity Feasible using Discriminant Function" IIT Delhi, India

[4] Samarasena Buchala, Neil Davey, Tim M. Gale, Ray J. Frank, "Principal Component Analysis of Gender, Ethnicity, Age, and Identity of Face Images," Department of Compute Science, University of Hertfordshire.

[5] F. Saei Manesh, M. Ghahramani, Y. P. Tan, "Facial Part Displacement Effect On Template based Gender and Ethnicity Classification," IEEE International Conference of Control, Automation, Robotics and Vision, pp. 1644-1649.

[6] Srinivas Gutta, Jeffrey R. J. Huang, P. Jonathon and, Harry Wechsler, "Mixture of Experts for Classification of Gender, Ethnic Origin, and Pose of Human Faces," IEEE Transaction on neural networks, Vol. 11, No.4, July 2000, pp. 948-960.

[7] Siyao Fu, Haibo He, Zeng-Guang Hou, "Learning Race from Face: A Survey," IEEE Transaction on Pattern Analysis and Machine Intelligence, Vol. 36, No.12, pp. 2483-2509.

[8] Md. Golam Moazzam, Mahbuba Begum, Mohammad Shorif Uddin, “Quantitative Analysis on Robustness of FLD and PCA-based Face Recognition Algorithms International Journal of Computer Application (0975 - 8887) Volume 99- No.19, August 2014,pp. 10-14

[9] Li Lu, Pengfei Shi, “A Novel Fusion-Based method for Expression- Invariant Gender Classification,” IEEE Transaction 2009. Pp. 1065-1068.

[10] Xiaoguang Lu, Hong Chen, and Anil K. Jain, "Multimodal Facial Gender and Ethnicity Identification," Michigan State University.

[11] Samarasena Buchala, Tim M, Gale, Neil Davey, Ray J. Frank, Kerry Foley, "Global And Feature Based Gender classification of Faces: A Comparasion of Human Performance And Computational Models," Department of Computer Science, University of Hertfordshire, pp. 1-10.

[12] Xiaoguang Lu, Anil K. Jain, "Ethnicity Identification from Face Images," ResearchGate, The International Society fo Optical Engineering, May 2004.

[13] M. Nazir, Muhammad Ishtiaq, Anab Batool, M. Arfan Jaffar, and Anwar M. Mirza., "Feature Selection for Efficient Gender Classification," In proc. WSEA international conference on Fuzzy systems, pp. 70-75, 2010.

[14] Omveer Singh, Gautam Bommagani, Sravan Reddy Ravula, Vinit Kumar Gunjan,"Pattern Based Gender Classification,” IJARCSSE, Volume 3, October 2013, pp. 888-895

[15] Ziyi Xu, Li Lu and Pengfei Shi., "A Hybrid Approach to Gender Classification from Face Images," in proc. IEEE International Conference on Pattern Recognition, pp. 1-4, 2008.

[16] S.Ravi, S.Wilson., "Face Detection with Facial Features and Gender Classification Based On Support Vector Machine." In proc. IEEE international conference on computational intelligence and computing research, pp. 23-28. 2010.

[17] Yingxiao Wu, Yan Zhuang, Xi Long, Feng Lin, Wenyao Xu, "Human Gender Classification:A Review,” IEEE Sensor Journal, July 2015, PP-1-12.

[18] Swathi Kalam, Geetha Guttikonda "Gender Classification using Geometric Facial Features," International Journal of computer Application(09758887), Volume 85-No 7, January 2014, pp. 32-37. 
[19] Li, B., et al., 2011. Gender classification by combining clothing, hair and facial component classifiers, Neurocomputing.

[20] Golomb, B., D. Lawrence and T. Sejnowski, 1990 Sexnet: A Neural Network identifies Sex from human faces, Advance in Neural Information Processing, Systems, pp: 572-577. 